\title{
Assessing the Therapeutic Potential of Targeted Attentional Bias Modification for Insomnia using Smartphone Delivery
}

Patrick J. F. Clarke ${ }^{1}$, Kristiina Bedford ${ }^{1}$, Lies Notebaert ${ }^{1}$, Romola S. Bucks ${ }^{1}$, Daniel Rudaizky ${ }^{1}$, Bronwyn C. Milkins ${ }^{1}$, Colin MacLeod ${ }^{1,2}$

${ }^{1}$ School of Psychology, University of Western Australia, Crawley, Western Australia, Australia

${ }^{2}$ School of Psychology, Babes-Bolyai University, Strada Mihail Kogãlniceanu 1, Mihail

Kogalniceanu St, Cluj-Napoca 3400, Romania.

Corresponding author: Patrick J. F. Clarke,

Email: patrick.clarke@uwa.edu.au

Mail: School of Psychology

University of Western Australia

35 Stirling Highway

Crawley 6009, Western Australia, Australia

Phone: +61 864887341

Fax: $\quad+61864881006$

Key words: Attentional bias; insomnia; attentional bias modification; cognitive bias modification; worry; anxiety; sleep. 
Cognitive models of insomnia propose that attentional vigilance for threat during the presleep period may play a causal role in insomnia by elevating physiological and psychological arousal to produce a state that is incompatible with the initiation of sleep [1]. Consistent with such theoretical accounts, studies have confirmed that insomnia is associated with patterns of biased attention favouring sleep-related threatening information [2].

Converging research has shown that attentional bias assessment methodologies, such as the attentional probe task, can be converted to attentional bias modification (ABM) tasks. Using such tasks it has been shown that modification of biased attention for threat can achieve therapeutic benefits in conditions where this bias is implicated, including anxiety and mood disorders [3]. Of specific relevance to the current research is the observation that a single session of computer-based ABM can have immediate effects on anxiety and stress reactivity [3]. Therefore, we sought to assess whether targeted delivery of an ABM task during the pre-sleep period could reduce symptoms of insomnia and the cognitive symptoms of pre-sleep arousal.

Invitations were extended to individuals from the University of Western Australia School of Psychology participant pool who had identified worry-related insomnia as a problem $(\mathrm{n}=236$, $\mathrm{n}=41$ accepted). Inclusion criteria (met by $\mathrm{n}=36$ ) were access to an iPhone, high levels of insomnia symptoms on the Pittsburgh Sleep Quality Index (PSQI $\geq 5$; [4]), completing at least four of five $\mathrm{ABM} / \mathrm{Control} \mathrm{sessions} \mathrm{during} \mathrm{the} \mathrm{study,} \mathrm{and} \mathrm{consistent} \mathrm{accuracy} \mathrm{on} \mathrm{the} \mathrm{attentional}$ probe task ( $>70 \%)$. Participants were randomly allocated to the ABM ( $\mathrm{n}=18,12$ female) or Control condition ( $\mathrm{n}=18,15$ female).

The study spanned eight days (seven nights). The first two days served as a baseline period in which participants completed self-report measures and wore the sleep monitor, but did not complete the ABM or control task. These initial baseline days were followed by five 
consecutive 'Task Completion Days' in which participants completed, immediately prior to bed, an $\mathrm{ABM}$ task (ABM condition) or a non-training control task (Control condition) on their smartphone. Both tasks employed 48 word-pairs comprising sleep-related threat words paired with non-threat words. The task format was similar to standard ABM tasks used in prior studies (see [3]), with task parameters adapted for use on an iPhone. On each trial an initial fixation cross was followed by two vertically aligned words, one threatening sleep-related word and one neutral word. After 500ms the word-pair was replaced by a probe appearing in the location of one of the words. Participants discriminated probe identity (horizontally or vertically aligned dots) by tapping the corresponding horizontal or vertical arrow icon at the bottom of the iPhone screen. In the ABM condition, probes always appeared opposite the threat word to encouraged attentional avoidance of sleep-related threat. In the Control condition and on assessment trials, probes replaced the threat and neutral words with equal frequency, so as not to encourage any pattern of selective attention. On Task Completion Days 1 to 5, participants completed 384 trials prior to sleep each night (approximately 15 minutes). Of these, 288 were ABM/Control trials and 96 were assessment trials randomly dispersed within the latter half of the ABM/Control trials. An index of attentional bias to threat was computed across assessment trials in a manner consistent with past studies (see [3]). Further details of task parameters, stimulus creation, experimental methodology, and additional discussion of findings are available in supplementary online material (http://www.psychology.uwa.edu.au/research/care/research/ClarkePPS).

On the first day of the study participants completed the trait version of the State-Trait Anxiety Inventory (STAI-T; [5]), the Penn State Worry Questionnaire (PSWQ; [6]) and the Pittsburgh Sleep Quality Index (PSQI; [4]), while baseline attentional bias was assessed with 96 trials of the attentional probe assessment task. On all days, participants completed the cognitive 
subscale of the Pre-Sleep Arousal Scale (PSAS-C; [7]) prior to sleep (following probe-task completion on Task Completion Days). Additionally, we assessed time taken to fall asleep (sleep onset latency), and frequency of waking both objectively, with a wireless electrophysiological monitor (EPSM; [8]), and subjectively via a sleep diary (see [9]). The latter also provided ratings of overall sleep quality (from 1-Very bad, to 5-Very good). Finally, changes in insomniarelated anxiety between Day 1 and 8 were assessed using the Anxiety and Preoccupation about Sleep Questionnaire (APSQ; [10]).

Participants in the ABM and Control Groups did not differ significantly in gender (p>.10), age, or any baseline measures, with the exception of sleep quality (see Table 1). All daily measures were subjected to an $\mathrm{ABM}$ condition (ABM/Control) by Probe Task Day (1-5) mixed model ANOVA. Results revealed main effects of ABM condition showing that, compared to those in the Control condition, those in the ABM condition reported significantly lower presleep arousal (on the PSAS-C), and better overall sleep quality (a reversal of the baseline difference). Additionally, EPSM measures showed that those in the ABM condition fell asleep faster and woke less often during the night compared to controls. No other significant main effects or interactions were observed (all $F<1.7$, all $p>.15$, see Table 1 for means and betweengroup differences).

Changes in sleep-related anxiety (APSQ) were assessed via a condition (ABM/Control) by assessment time (pre/post-assessment) mixed model ANOVA, which showed a significant interaction effect, $F(1,34)=5.44, p=.03, \eta^{2}=.16$. This interaction was carried by a significant decrease in APSQ scores from pre-assessment $(\mathrm{M}=61.61, \mathrm{SD}=18.00)$ to post-assessment $(\mathrm{M}=40.72, \mathrm{SD}=13.38)$ in the $\mathrm{ABM}$ group, $t=3.86, p<.01, d=1.32$, that did not approach significance for the pre-assessment $(\mathrm{M}=61.50, \mathrm{SD}=17.88)$ to the post-assessment $(\mathrm{M}=56.17$, 
$\mathrm{SD}=14.34)$ in the Control group, $t=1.36, p=.19, d=0.33$. In summary, as compared to the Control condition, participants who completed ABM training reported less pre-sleep arousal, fell asleep faster, woke less often during the night, reported better overall sleep quality, and showed significant reductions in sleep-related anxiety from before to after the five-day program.

Although these effects are entirely consistent with the positive effects of ABM on relevant sleep measures, we did not also observe significant change in attentional bias. While this may represent a genuine absence of bias change, the consistency of effects across separate sleep measures suggests that $\mathrm{ABM}$ was having the intended effect. It is possible therefore that iPhonebased assessment coupled with the relatively small sample size may not have been sufficient to detect bias changes. Indeed, a post-hoc power analysis suggests that in order to detect a medium sized between-group effect in attentional bias it would be necessary to recruit a sample with more than 50 participants.

Overall, these results suggest that attentional bias modification targeting vigilance for sleep-related threat during the pre-sleep period has the capacity to reduce cognitive arousal and improve insomnia symptoms, providing a crucial step towards establishing a novel intervention for insomnia. Given the small sample size, replication with a larger sample will be crucial before it is possible to draw firm conclusions about potential benefits of ABM for insomnia.

Nevertheless the results provide encouragement that targeted ABM could be used not only as a potential treatment for insomnia, but also with other conditions that implicate attentional bias (and consequent anxious arousal), as being acutely problematic at specific points in time. These findings therefore not only highlight a novel target for ABM in insomnia, but also a novel method of ABM by targeting acute rather than enduring change in attentional bias. 


\section{Acknowledgments}

PJFC is supported by an Australian Research Council Grant DP140103713, CM is supported by an Australian Research Council Grant DP140104448, and a grant from the Romanian National Authority for Scientific Research CNCS-UEFISCDI, Project No. PNII-IDPCCE-2011-2-0045.

\section{Disclosure Statement}

All authors report no conflicts of interest. 
Table 1. Comparison of participant characteristics, mean baseline daily measures, and mean Probe Task daily measures between active $\mathrm{ABM}$ and control groups.

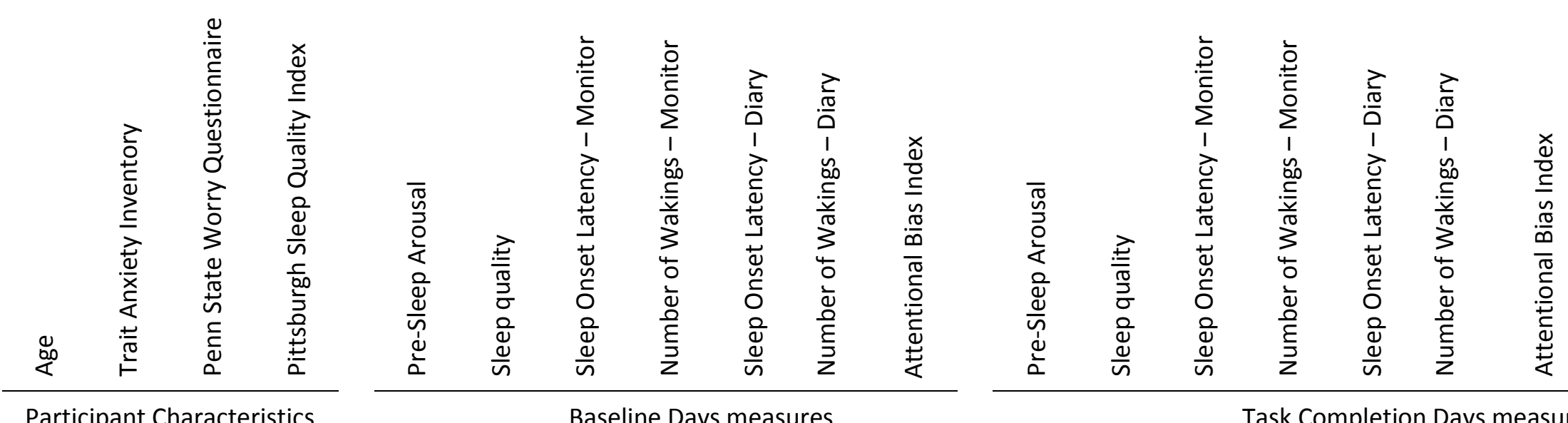

\begin{tabular}{|c|c|c|c|c|c|c|c|c|c|c|c|c|c|c|c|c|c|c|}
\hline & 品 & 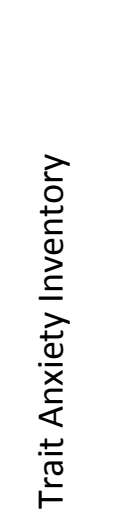 & 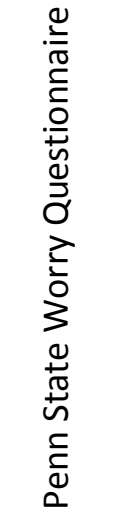 & 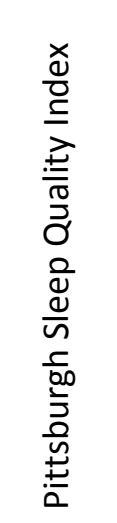 & 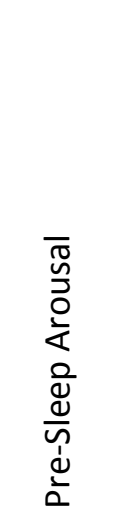 & 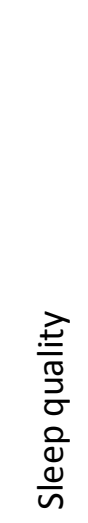 & 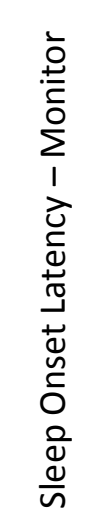 & 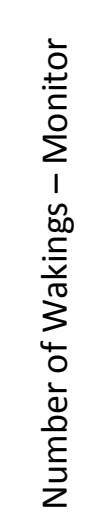 & 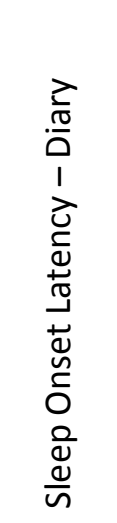 & 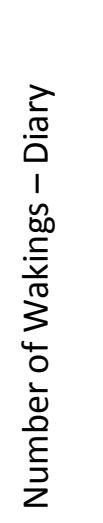 & 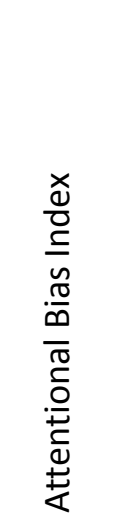 & 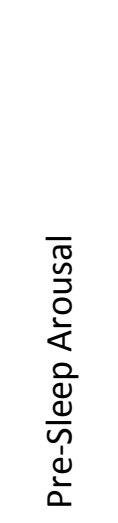 & 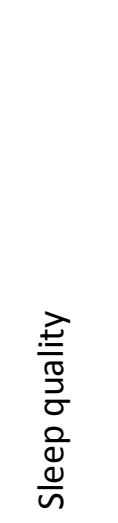 & 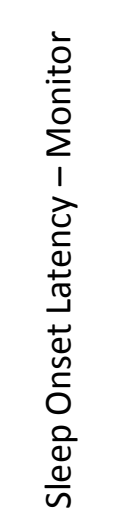 & 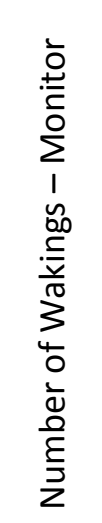 & 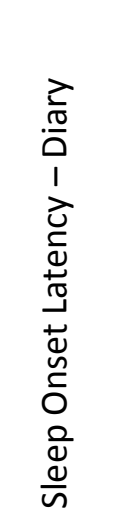 & 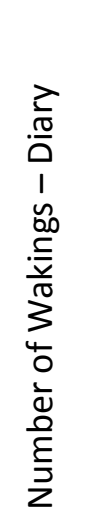 & 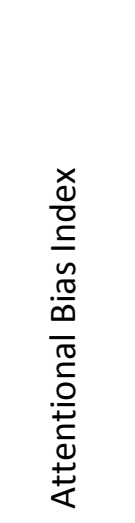 \\
\hline & \multicolumn{4}{|c|}{ Participant Characteristics } & \multicolumn{7}{|c|}{ Baseline Days measures } & \multicolumn{7}{|c|}{ Task Completion Days measures } \\
\hline \multicolumn{19}{|l|}{ ABM Condition } \\
\hline$M$ & 19.89 & 50.00 & 51.11 & 18.16 & 20.94 & 2.47 & 41.46 & 2.29 & 28.94 & 1.94 & -14.8 & 17.56 & 3.43 & 31.34 & 1.76 & 29.50 & 1.42 & 7.65 \\
\hline$S D$ & 2.95 & 8.68 & 6.07 & 11.64 & 4.59 & 0.55 & 25.17 & 1.49 & 15.57 & 1.40 & 22.8 & 6.66 & 0.77 & 22.70 & 1.07 & 31.40 & 1.24 & 29.64 \\
\hline \multicolumn{19}{|c|}{ Control Condition } \\
\hline M & 18.33 & 54.72 & 53.78 & 14.00 & 24.44 & 3.06 & 46.00 & 2.65 & 36.49 & 1.76 & -5.01 & 22.46 & 2.91 & 53.48 & 3.05 & 39.22 & 1.16 & -0.58 \\
\hline$S D$ & 2.06 & 8.36 & 8.21 & 6.87 & 6.49 & 0.59 & 32.99 & 2.46 & 26.07 & 1.13 & 27.66 & 7.63 & 0.95 & 42.95 & 2.07 & 42.55 & 1.04 & 19.01 \\
\hline \multicolumn{19}{|l|}{ Comparison } \\
\hline$t$ & 1.83 & 1.66 & 1.11 & 1.31 & 1.87 & 3.08 & 0.46 & 0.53 & 1.05 & 0.50 & 1.17 & 2.45 & 3.34 & 2.65 & 2.34 & 1.02 & 0.69 & 0.99 \\
\hline$p$ & .08 & .11 & .28 & .20 & .07 & $.01^{*}$ & .64 & .60 & .29 & .68 & .25 & $.02 *$ & $<.01^{*}$ & $.01^{*}$ & $.02 *$ & .31 & .49 & .33 \\
\hline$d$ & 0.61 & 0.55 & 0.37 & 0.44 & 0.62 & 1.03 & 0.15 & 0.18 & 0.35 & 0.14 & .39 & 0.68 & 0.60 & 0.64 & 0.78 & .26 & 0.23 & 0.33 \\
\hline
\end{tabular}




\section{References}

1 Espie CA, Broomfield NM, MacMahon K, Macphee LM, Taylor LM: The attentionintention-effort pathway in the development of psychophysiologic insomnia: A theoretical review. Sleep Medicine Reviews 2006;10:215-245.

2 Harris K, Spiegelhalder K, Espie CA, MacMahon KMA, Woods HC, Kyle SD: Sleeprelated attentional bias in insomnia: A state-of-the-science review. Clinical Psychology Review 2015

3 MacLeod C, Clarke PJF: Cognitive bias modification: A new frontier in cognition and emotion research; in Robinson MD, Watkins ER, Harmon-Jones E (eds): Handbook of cognition and emotion. New York, Guilford Press, 2013, pp 540-562.

4 Buysse DJ, Reynolds Iii CF, Monk TH, Berman SR, Kupfer DJ: The pittsburgh sleep quality index: A new instrument for psychiatric practice and research. Psychiatry research 1989;28:193-213.

$5 \quad$ Spielberger C, Gorsuch R, Lushene R, Vagg P, Jacobs G: Manual for the state-trait anxiety inventory stai (form y): Self-evaluation questionnaire. Alto: Ca, Consulting Psychologist Press., 1983.

6 Meyer T, Miller M, Metzger R, Borkovec TD: Development and validation of the penn state worry questionnaire. Behaviour Research and Therapy 1990;28:487-495.

$7 \quad$ Nicassio PM, Mendlowitz DR, Fussell JJ, Petras L: The phenomenology of the pre-sleep state - the development of the pre-sleep arousal scale. Behaviour Research and Therapy 1985;23:263-271.

8 Shambroom JR, Fabregas SE, Johnstone J: Validation of an automated wireless system to monitor sleep in healthy adults. Journal of Sleep Research 2012;21:221-230.

9 Morin CM: Insomnia: Psychological assessment and management. New York, Guilford Press, 1993.

10 Tang NKY, Harvey AG: Correcting distorted perception of sleep in insomnia: A novel behavioural experiment? Behaviour Research and Therapy 2004;42:27-39. 


\title{
Assessing the Therapeutic Potential of Targeted Attentional Bias Modification for Insomnia Using Smartphone Delivery
}

\author{
Patrick J.F. Clarke, Kristiina Bedford, Lies Notebaert, Romola S. Bucks, Daniel Rudaizky, Bronwyn C. \\ Milkins, Colin MacLeod
}

\section{Supplementary Online Material}

\section{Creation of experimental word stimuli}

Research assessing the presence of attentional bias in insomnia has consistently employed paired word stimuli that contain a threatening member, which communicates a meaning relevant to the concerns of those with sleep disturbance, and an emotionally neutral stimulus member (see [1]). In line with this, we included 48 threatening sleeprelated words characteristic of pre-sleep worry thoughts, paired with neutral words matched for length and spoken frequency [2] for the purpose of modification and assessment of attentional bias. The threat words were selected from an initial pool of 96 candidate words. These words were generated on the basis of three factors identified by Wicklow and Espie [3] as core themes relevant to presleep worry consistently reported by individuals with insomnia: active problem solving (e.g. thoughts about the negative consequences of poor sleep), present-state monitoring (e.g. thoughts about bodily functions), and reactivity to external stimuli (e.g. thoughts about environmental factors disturbing sleep). All 96 candidate words were rated by 12 independent judges using a 7-point scale on emotional valence, from 1 (Very negative) to 7 (Very positive) and relatedness to sleep concerns, from 1 (Not at all) to 7 (Extremely). The final selected threat words were rated to be strongest in negative valence $(M=2.35$, $\mathrm{SD}=0.78)$ and most relevant to sleep concerns $(M=4.67$, $\mathrm{SD}=0.93$ ). The word pairs were randomised and divided into two separate stimulus subsets (Subset A and Subset B). For half the participants in each condition Subset A was used in the training/control component and Subset B was used in the assessment component of the task, while the remaining participants received the reverse allocation. The use of different stimuli in the training and assessment components ensures that any change in attentional bias can be attributed to the class of stimulus and not to specific stimuli themselves. These stimuli are provided in Supplementary Table 1.

\section{Probe task parameters}

We sought to use an attentional probe training paradigm as this type of task has been successfully employed in past research to modify patterns of biased attention for threat $[4,5]$, and it has been used to establish the presence of attentional bias for threat in insomnia [1]. Trials on the probe task all shared a common structure. Each trial began with a white fixation cross $(+)$ on a black background presented in the center of the screen for $500 \mathrm{~ms}$. Two words then appeared in white text for $500 \mathrm{~ms}$, one threat and one neutral, vertically aligned and separated by $25 \mathrm{~mm}$. Immediately following the termination of this display a visual probe comprising two small red dots Supplementary Table 1. Word stimuli employed in the attentional probe task. The threat word is the first member of each pair

Stimulus Word Pairs

\begin{tabular}{|c|c|}
\hline Subset A & Subset B \\
\hline Ache $\neq$ - Melt & Lazy $\neq-$ Hint \\
\hline Afraid $¥$ - Artist & Lethargy* - Teaspoon \\
\hline Agitated* - Carriers & Medication - Bottleneck \\
\hline Alarm - Spray & Inept - Sedan \\
\hline Angrył - Curve & Nightmare $\neq$ - Volunteer \\
\hline Anxiety $\neq$ - Express & Overactive $\neq$ - Clearances \\
\hline Confuse - Drawers & Panickył - Infuser \\
\hline Conflict $\neq$ - Advanced & Restless $\neq$ - Receiver \\
\hline Delirious $\ddagger$ - Fragments & Sad $\neq$ - Cup \\
\hline Desperate $\neq$ - Aesthetic & Sick* - Pull \\
\hline Disastrous $\ddagger$ - Curriculum & Sleepless* - Advisable \\
\hline Disengaged $\neq$ - Nonfiction & Sleepy* - Wallet \\
\hline Disorder $\neq$ - Compost & Sluggish $\neq$ - Decorate \\
\hline Distract $\neq$ - Brochure & Snoring $\neq$ - Charity \\
\hline Distress $\ddagger$ - Creature & Stress* - Links \\
\hline Drowsy* - Umpire & Suffer $\neq$ - Branch \\
\hline Exhausted* - Developed & Tense $\neq$ - Trick \\
\hline Fatigue* ${ }^{*}$ Passive & Time $\neq$ - Look \\
\hline Hopeless $\ddagger$ - Borrowed & Tired $\neq$ - Plain \\
\hline Illness* - Mustard & Unpleasant $\neq$ - Expedition \\
\hline Inadequate $\neq$ - Initiative & Wakefulł - Trolley \\
\hline Irrationalł - Flashlight & Work* - Life \\
\hline Irritable $\neq$ - Appliance & Workload* - Handbags \\
\hline Jittery $\ddagger$ - Thermos & Worrył - Theme \\
\hline
\end{tabular}

*Active problem-solving, $\ddagger$ Present-state monitoring, $\sim$ Environmental reactivity [cf. 3]

oriented either horizontally (..) or vertically (:) appeared in the location vacated by one of the words. Threat word position (upper/lower) and probe type (horizontal/vertical probe) was randomised within the constraint that an equal number of each probe type was presented, and the threat word appeared equally often in the upper and lower position. Participants discriminated probe identity by tapping the corresponding horizontal or vertical arrow icon at the bottom of the display (see Figure 1).

Across the 384 trials completed on Task Completion Days 1-5 before bed each night, for 288 trials (12 repetitions of 24 words pairs), the position of the probe was contingent on the allocated condition. For those in the ABM condition, visual probes consistently replaced neutral words to 


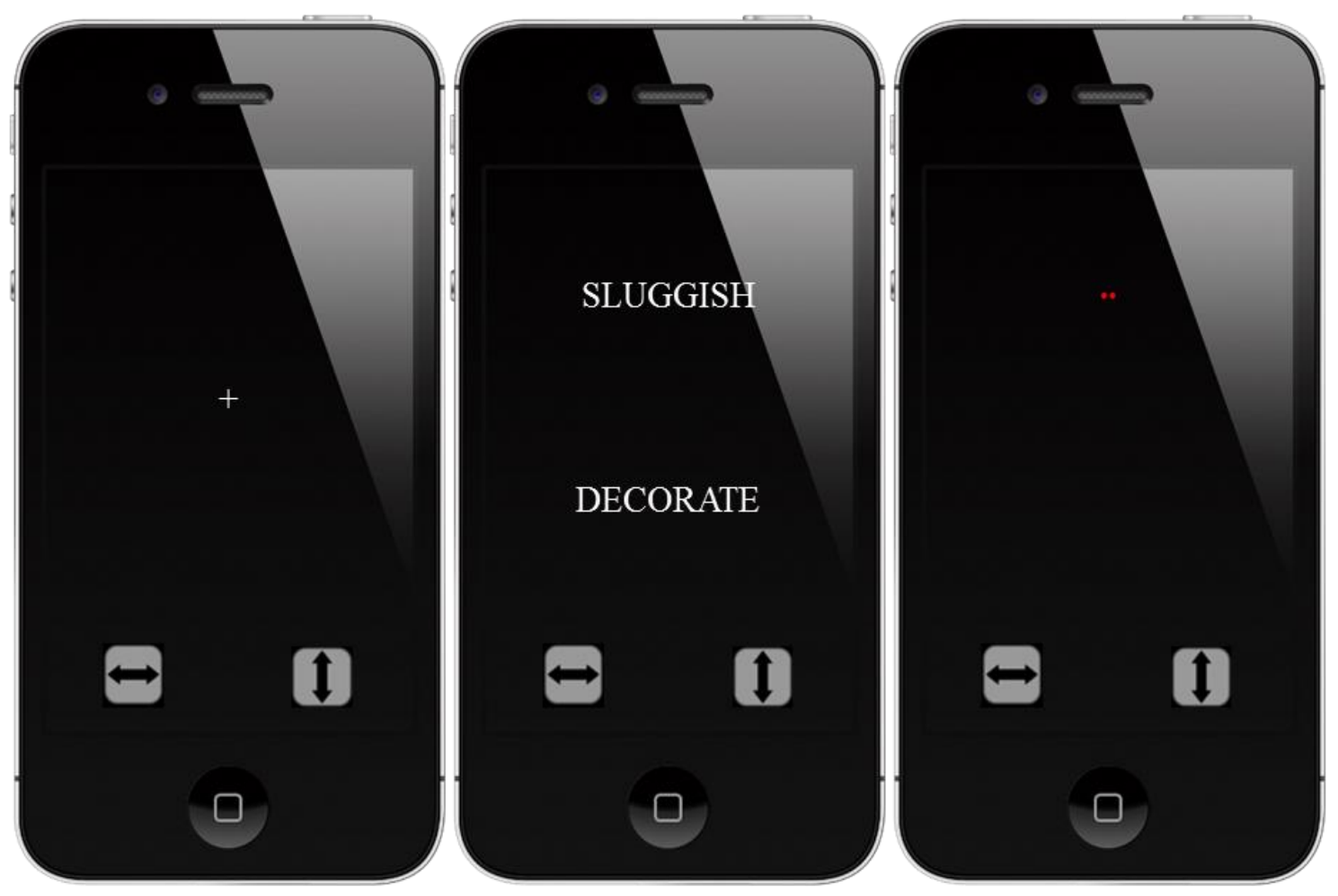

Fig 1. Example trial of the attentional probe task delivered via iPhone.

encourage attentional avoidance of threat. Conversely, for those in the control condition, the task was identical in all aspects with the exception that visual probes replaced threat and neutral words with equal frequency on each trial. The remaining 96 trials in each condition (4 repetitions of 24 words pairs) were attentional assessment trials where probes replaced the threatening and neutral member of word pairs with equal frequency. While a continuous block of attentional assessment trials permits a discrete period of attentional assessment, there is also the possibility that such a discrete block of trials can potentially disrupt/compromise an acquired pattern of attentional selectivity and any associated effects. As such, and consistent with the approach of a number of previous studies [e.g. 6, 7, 8] assessment trials were incorporated into the training trials by randomly dispersing the 96 attentional assessment trials in the latter half of the training trials. Reaction time data derived from the attentional probe assessment trials was prepared in a manner consistent with previous studies [9, 10]. Specifically, to limit the influence of outlying data, trials with incorrect responses (4.16\% of trials) or latencies less than $200 \mathrm{~ms}(0.25 \%$ of trials $)$ or greater than $2000 \mathrm{~ms}$ $(1.52 \%$ of trials) were removed.

\section{Electrophysiological sleep monitor}

We employed a commercially available electrophysiological sleep monitoring system (EPSM; Zeo, Inc., Newton, MA, USA). The EPSM uses dry silver-coated fabric to collect electrophysiological signals from the forehead with a single bi-polar channel located at approximately Fp1-Fp2 [11]. Research comparing sleep measures derived from this EPSM device with polysomnography has revealed a high degree of concordance, particularly in relation to sleep and wakefulness measures [92.6\% and $91.1 \%$ respectively; 12$]$.

\section{Experimental Procedure}

Prior to the commencement of the study, participants were told only that the study sought to examine the relationship between sleep disturbance, worry, and patterns of cognitive information processing and were not alerted to the presence of the alternative experimental conditions. The attentional probe task application was installed on participants' iPhones, along with links to the pre-sleep and sleep diary questionnaire measures. Participants were given detailed instructions on how to access these measures and how to complete the attentional probe task. They then completed 96 attentional probe assessment trials. Lastly, participants were provided instructions in the use of the electrophysiological sleep monitor (EPSM) and observed the correct way to fit the device, charge the headband, and how to set up the bedside base station. Participants were instructed that on all days, prior to sleep each night they should complete the pre-sleep questionnaire via the app, wear the EPSM from the moment they intended to go to sleep until morning, and upon waking update their daily sleep diary. In addition, they were instructed to complete the attentional probe task prior to the pre-sleep questionnaire measure each night from the third to the final day (Task Completion Days 1-5). At the follow-up session (8 days after the pre-assessment) participants completed the postassessment questionnaire measures, the EPSM system was returned, and the attentional probe task application was removed from participants' iPhones. In addition, participants were debriefed about the purpose and design of the study. 


\section{Results}

The following includes graphical representations of key findings described in the main manuscript.
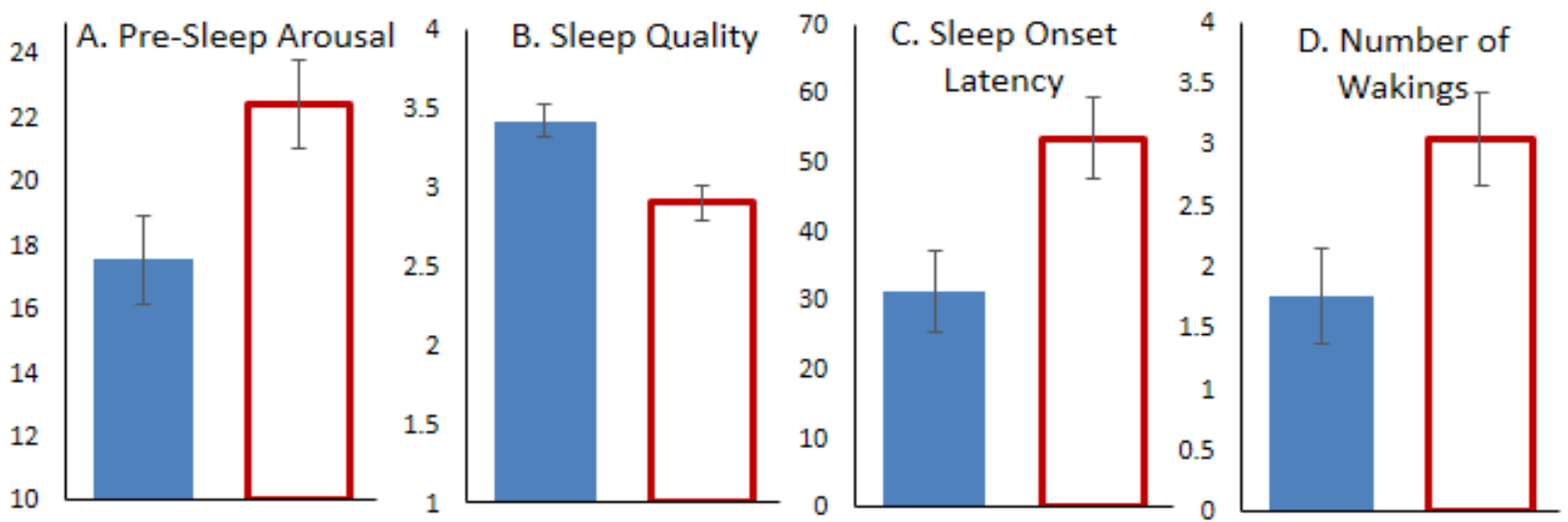

Attention Bias Modification $\quad$ CControl

Fig 2. Daily sleep measures for participants in the Attentional Bias Modification and Control conditions showing group differences on the pre-sleep arousal scale (A: $t=2.45, p=.02, d=0.68)$, self-rated sleep quality (B: $t=3.34, p<.01, d=0.60)$, sleepmonitor assessed sleep onset latency in minutes $(\mathrm{C}: t=2.65, p=.01 . d=0.64)$ and sleep-monitor assessed number of wakings (D: $t=2.34, p=.02, d=0.78$ ).

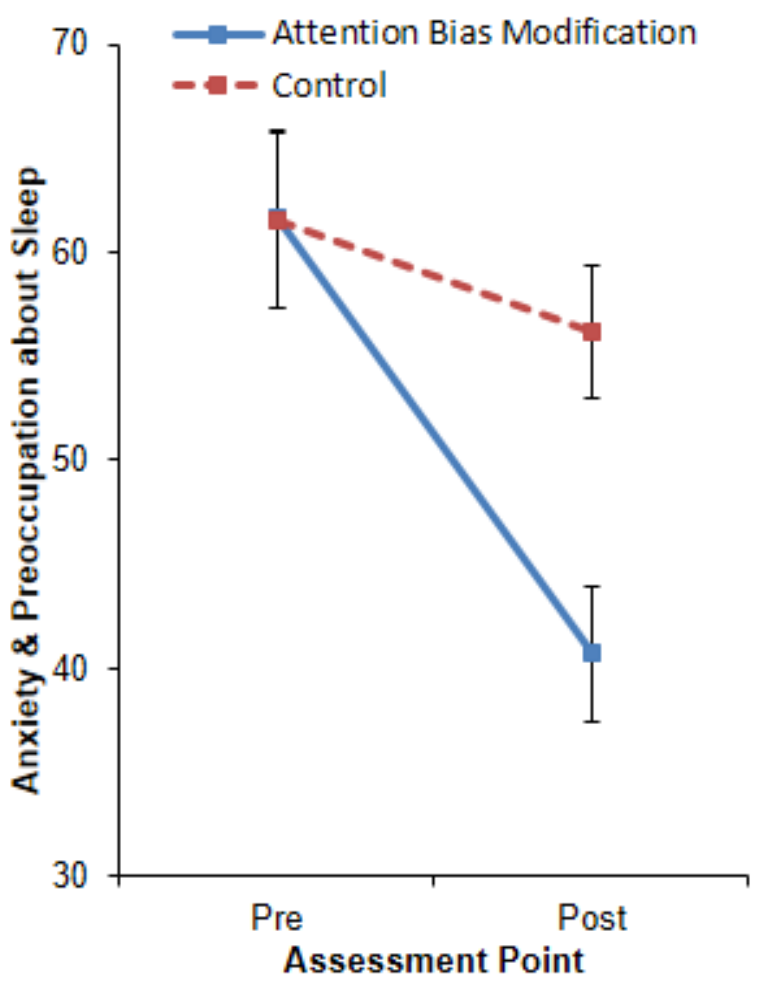

Fig 3. Change in sleep-related anxiety from preassessment to post-assessment for the Attentional Bias Modification and Control groups. Pre-assessment between group comparison: $t=0.02, p=.98, d=0.01$; postassessment between group comparison $t=3.34, p<.01$, $d=1.11$.

\section{Supplementary Discussion \\ Assessment of Attentional Bias}

Unlike measures of sleep quality, pre-sleep arousal, sleep onset latency, and number of wakings, we did not also observe consistent change in measures of attentional bias in line with each condition. While it is possible that this pattern of findings represents a genuine absence of bias change, the consistent pattern of effects across a number of separate measures suggests that $\mathrm{ABM}$ was having the intended effect on sleep and arousal. These effects therefore provide support for the alternative possibility, that the intended bias change may have been achieved but not detected.

Of relevance to this is the fact that attentional bias was assessed using smartphones. The use of smartphones in the current study provided a convenient and highly accessible method of delivering $\mathrm{ABM}$ at the precisely intended time. However, greater flexibility in task delivery comes at the potential expense of greater precision for assessing attentional bias. ABM induced changes in attentional bias commonly occur within the range of 10-20 milliseconds [9, 13]. Computer-based tasks delivered in laboratory settings allow for precise control across a range of factors relevant to the assessment of attentional bias (e.g. stimulus viewing distance, reaction time measurement, etc.). As smartphones have not been validated as a means of assessing patterns of biased attention, this could potentially account for our failure to confirm attentional bias change in line with the assigned attentional training condition. To permit more precise assessment of attentional bias future research could employ pre and post-intervention computer-based assessments to confirm changes in attentional bias. However, it is possible that if the attention bias modification task resulted in only very acute, rather than enduring change in attentional bias for sleep-related threat, then lab-based assessment may not show a between-group change in 
attentional bias, even if such a change is achieved using the iPhone ABM task. For this reason it may be ideal to include a comparison computer-based pre-sleep training and assessment of attentional bias to confirm change in attentional bias in response to the ABM task.

\section{Effects on Self-Report and Object Sleep Measures}

While the positive impact of ABM was demonstrated on objective measures obtained from sleep monitors and from participants' ratings of sleep quality in general, there was no observed effects on self-report measures of sleep onset or frequency of waking. However, as previously noted, the tendency towards misperception of sleep outcomes characteristic of poor sleepers [14] means that such self-report measures requiring retrospective estimates of the time taken to lose consciousness, or the frequency of waking, are unlikely to be reliable indices of sleep. Indeed, the presence of significant effects on both objective measures obtained from sleep monitors, and on self-report measures of arousal and sleep quality provides encouragement that $\mathrm{ABM}$ was having the intended effect. These findings are particularly noteworthy given the brief implementation of $\mathrm{ABM}$ and the non-pharmacological nature of the intervention, and suggest that the ABM procedure was successful in briefly reducing cognitive arousal symptoms, allowing greater ease of sleep onset and more restful sleep.

\section{Supplementary References}

1. Harris, K., et al., Sleep-Related Attentional Bias in Insomnia: A State-of-the-Science Review. Clinical Psychology Review, 2015.

2. Wilson, M., MRC Psycholinguistic Database: Machineusable dictionary, version 2.00. Behavior Research Methods, Instruments, \& Computers, 1988. 20(1): p. 610.

3. Wicklow, A. and C. Espie, Intrusive thoughts and their relationship to actigraphic measurement of sleep: towards a cognitive model of insomnia. Behaviour research and therapy, 2000. 38(7): p. 679-693.

4. Amir, N., et al., Attention modification program in individuals with generalized anxiety disorder. Journal of Abnormal Psychology, 2009. 118(1): p. 28-33.

5. MacLeod, C., et al., Selective attention and emotional vulnerability: Assessing the causal basis of their association through the experimental manipulation of attentional bias. Journal of Abnormal Psychology, 2002. 111(1): p. 107-123.

6. Krebs, G., C.R. Hirsch, and A. Mathews, The effect of attention modification with explicit vs. minimal instructions on worry. Behaviour Research and Therapy, 2010. 48(3): p. 251-256.

7. Wells, T.T. and C.G. Beevers, Biased attention and dysphoria: Manipulating selective attention reduces subsequent depressive symptoms. Cognition and Emotion, 2010. 24(4): p. 719-728.

8. Hayes, S., C.R. Hirsch, and A. Mathews, Facilitating a benign attentional bias reduces negative thought intrusions. Journal of Abnormal Psychology, 2010. 119(1): p. 235-240.

9. Amir, N., et al., Attention training in individuals with generalized social phobia: A randomized controlled trial. Journal of Consulting and Clinical Psychology, 2009. 77(5): p. 961-973.

10. Clarke, P.J.F., et al., The Causal Role of the Dorsolateral Prefrontal Cortex in the Modification of Attentional Bias: Evidence from Transcranial Direct Current Stimulation. Biological Psychiatry, 2014. 76(12): p. 946-952.

11. Kelly, J.M., R.E. Strecker, and M.T. Bianchi, Recent developments in home sleep-monitoring devices. ISRN neurology, 2012. 2012: p. 768794-768794.

12. Shambroom, J.R., S.E. Fabregas, and J. Johnstone, Validation of an automated wireless system to monitor sleep in healthy adults. Journal of Sleep Research, 2012. 21(2): p. 221-230.

13. Amir, N., et al., The effect of a single-session attention modification program on response to a public-speaking challenge in socially anxious individuals. Journal of Abnormal Psychology, 2008. 117(4): p. 860-868.

14. Means, M.K., et al., Accuracy of sleep perceptions among insomnia sufferers and normal sleepers. Sleep Medicine, 2003. 4(4): p. 285-296. 